\title{
Evaluating TCMS Train-to-Ground communication performances based on the LTE technology and discreet event simulations
}

Bouaziz, Maha; Yan, Ying; Kassab, Mohamed; Soler, José; Berbineau, Marion

Published in:

Proceedings of the 13th International Workshop on Communication Technologies for Vehicles

Link to article, DOI:

10.1007/978-3-319-90371-2_12

Publication date:

2018

Document Version

Publisher's PDF, also known as Version of record

Link back to DTU Orbit

Citation (APA):

Bouaziz, M., Yan, Y., Kassab, M., Soler, J., \& Berbineau, M. (2018). Evaluating TCMS Train-to-Ground communication performances based on the LTE technology and discreet event simulations. In Proceedings of the 13th International Workshop on Communication Technologies for Vehicles (pp. 110-121). Springer. Lecture Notes in Computer Science Vol. 10796 https://doi.org/10.1007/978-3-319-90371-2_12

\section{General rights}

Copyright and moral rights for the publications made accessible in the public portal are retained by the authors and/or other copyright owners and it is a condition of accessing publications that users recognise and abide by the legal requirements associated with these rights.

- Users may download and print one copy of any publication from the public portal for the purpose of private study or research.

- You may not further distribute the material or use it for any profit-making activity or commercial gain

- You may freely distribute the URL identifying the publication in the public portal 


\title{
Evaluating TCMS Train-to-Ground communication performances based on the LTE technology and discreet event simulations
}

\author{
Maha Bouaziz ${ }^{1}$, Ying Yan $^{2}$, Mohamed Kassab $^{3}$, José Soler ${ }^{2}$, Marion Berbineau $^{1}$ \\ ${ }^{1}$ University Lille Nord de France, IFSTTAR, COSYS, Villeneuve d'Ascq, F-59650 \\ ${ }^{2}$ Technical University of Denmark, 2800 Kgs.Lyngby, Denmark \\ ${ }^{3}$ Laboratory NOCCS, University of Sousse, Tunisia \\ maha.bouaziz@ifsttar.fr
}

\begin{abstract}
This paper focuses on performance evaluation of the Train to Ground traffic exchanges used to ensure safety and monitoring train functionalities carried by the Train Control Management System (TCMS). In the framework of the European project Safe4Rail from the Shift2Rail program, we try to use LTE (Long Term Evolution) network as an alternative communication technology, instead of GSM-R (Global System for Mobile communications-Railway) because of some capacity and capability limits. First step, a pure simulation is used to evaluate the network load for a high-speed scenario, when the LTE network is shared between the train and different passengers. The simulation is based on the discrete-events network simulator Riverbed Modeler. Next, second step focusses on a co-simulation testbed, to evaluate performances with real traffic based on Hardware-In-The-Loop and OpenAirInterface modules. Preliminary simulation and co-simulation results show that LTE provides good performance for the TCMS traffic exchange in terms of packet delay and data integrity.
\end{abstract}

Keywords: TCMS, discreet event simulator, LTE, Riverbed, OpenAirInterface, Railway, Mobile Communication Gateway, Train-to-ground communication.

\section{Introduction}

Train Control \& Management System (TCMS) is a train-borne distributed control system. It provides data communications interfaces to other train-borne systems, and also telecommunications to support systems operating remotely on the wayside. TCMS is often referred to as the "brain of the train" due to its central role in coordinating control and monitoring across disparate systems [1]. The data for TCMS services can be safety-related or not. The onboard network carries operational services like CCTV (Close Circuit TeleVision), passenger information, etc. The services are also customeroriented such as access to Internet, infotainment... In order to reduce operation and maintenance costs in the railway domain, wired communication networks are replaced by wireless systems [2-3]. The communication between TCMS and ground systems is provided through the so-called Mobile Communication Gateway (MCG) and the 
Ground Communication Gateway (GCG), as specified in IEC61375 [4]. These two entities are under development in the Shift2Rail CONNECTA project [5] and they will be able to connect themselves to several types of public wireless systems such as WiFi or LTE for example. In this context, it is very important to develop various simulation/emulation tools that can allow the evaluation of the system with a "zero on site testing" approach promoted in the Shift2rail program.

As described in the EU project Safe4rail [6-7], we are developing a hardware and software simulation platform using OpenAirInterface and Riverbed Modeler for Railway Communication that is able to evaluate TCMS performance while varying the railway environment and the use cases. Based on the test environment design in [8], we consider, in this paper, a LTE network deployed along a railway line and we present preliminary results using the hardware and software platform and the pure simulated network. We focus on the high-speed rail context.

\section{Pure Simulation Platform}

Several research works, in the literature, deal with field tests considering LTE signal. Several authors have also considered discrete-event simulator such as Riverbed and have evaluated performances of LTE-based train-to-ground communication in the context of Control and command with a dedicated LTE network that replace a GSM-R one or CBTC (Communication Based Train Control) [9-11].

In this Section, we consider a LTE network simulation based on a network scenario defined in [12], which uses a trajectory line covered by regular LTE Macro-cells. The TCMS traffic performances are evaluated regarding the end-to-end delay and the data loss indicators.

The test environment consists of a train and various passengers. The train is moving and exchanging TCMS traffic with ground devices (GCG) through the LTE wireless access, whereas passengers are using different applications and sharing the same network. The goal of this scenario is to evaluate the efficiency of LTE QoS management, when TCMS traffic shares the network access with other traffics. The network load is increased by different traffics from the User Equipment (UE) representing the train passengers.

\subsection{Simulation topology and parameters}

We consider the discrete-events network simulator called Riverbed Modeler (formerly OPNET Modeler ${ }^{\circledR}$ ) V.18.6.0 (Release Oct 31, 2016) to perform the proposed evaluations [13-14]. Riverbed enables to model the network architecture of the test environment, the TCMS applications and the passenger UE applications. In addition, it is used to evaluate the interaction and the data exchanges between all network entities.

The scenario models a railway line in Denmark, between Snoghoj and Odense [12]. This line length is about $54.5 \mathrm{~km}$. In the Riverbed simulation environment, the coverage of this line needs the deployment of $11 \mathrm{eNodeB}$ (eNBs) in the $900 \mathrm{MHz}$ band as illustrated in Fig. 1, where a train is required to perform multiple handover in moving with 
$300 \mathrm{~km} / \mathrm{h}$. eNBs are connected to the Evolved Packet Core (EPC) equipment, which provides the connectivity with the GCG equipment.

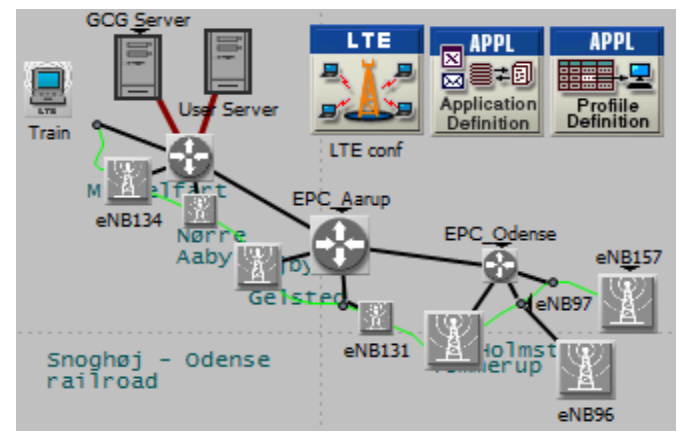

Fig. 1. Topology architecture of the Snoghoj-Odense railway trajectory.

LTE parameters. Our model is based on the Riverbed LTE model. The train and passengers are modeled as LTE User Equipments (UE). eNBs are connected to an EPC node, which models the whole LTE backbone functionalities. Then, the TCMS application servers and passenger application servers are directly connected to the EPC node. Regarding the LTE radio coverage, we propose a basic macro cell based coverage for the high-speed scenario, since macro cells are more suitable for this scenario kind [1516]. Table 1 summarizes the used LTE parameters.

Table 1. LTE configuration parameters [14].

\begin{tabular}{ll}
\hline Parameter & Macro cells \\
\hline Frequency band & $920 \mathrm{MHz}(\mathrm{BW}: 5 \mathrm{MHz})$ \\
eNB Transmission power & $36 \mathrm{dBm}$ \\
eNB antenna height & 50 meters \\
eNB antenna gain & $15 \mathrm{dBi}$ \\
UE antenna gain & $1 \mathrm{dBi}$ \\
Pathloss model & $\mathrm{UMa}^{1}$ \\
Multipath channel model & ITU Pedestrian $\mathrm{A}^{2}$ \\
\hline
\end{tabular}

1 : ITU-R M2135 Urban Macro (UMa). The simulation randomly chooses between Line-of-Sight and Non-Line-of-Sight cases. This parameter was chosen for a preliminary configuration.

2: The ITU Pedestrian A multipath channel model is chosen following parameters used in [12], for a preliminary configuration. This model will be changed after validation of the simulations principle.

Application parameters. Based on the European Roll2Rail project [17], the TCMS traffic is varied following different uses cases [2]. In the Safe4Rail project, we have selected traffic types that represent the highest constraints: signaling data, video data, 
file date update and voice data as described in Table 2. These traffics are considered for the evaluation.

Table 2. TCMS application modeling

\begin{tabular}{lll}
\hline Application & Datagram Size & Data Rate \\
\hline Signalling data & 128 bytes & UL 8 kbps / DL 4.6 kbps \\
Video data & 6250 bytes & UL 1 Mbps \\
Voice data (live) & 160 bytes & UL 64 kbps \\
Voice data (recorded) & 160 bytes & DL 64 kbps \\
File data & 1000 bytes & UL 2 Mbps \\
\hline
\end{tabular}

A set of basic user profiles predefined by Riverbed Modeler models the passenger traffic. Each profile defines a set of usual LTE user applications that exchanges traffic with servers in the core network. The passenger UE profiles are presented in Table 3.

Table 3. Passenger UE profiles and their traffics

\begin{tabular}{ll}
\hline User Profile & Traffics \\
\hline Mobile User (MoU) & Instant messaging, Gaming, Interactive content Pull \\
Multimedia User (MuU) & VoIP and video conferencing \\
Engineer User (EnU) & Web browsing, Email, Telnet session, File transfer \\
\hline
\end{tabular}

Table 4. LTE bearer setting

\begin{tabular}{llll}
\hline EPS bearer & Signalling & Voice & Default \\
\hline Application(s) & $\begin{array}{l}\text { Signalling } \\
\text { data }\end{array}$ & Voice data & Other \\
QoS Class Identifier (QCI) & $3(\mathrm{GBR})$ & $2(\mathrm{GBR})$ & 9 (Non-GBR) \\
Guaranteed bitrate (uplink) & $16 \mathrm{kbps}$ & $64 \mathrm{kbps}$ & - \\
Guaranteed bitrate (down.) & $16 \mathrm{kbps}$ & $64 \mathrm{kbps}$ & - \\
Allocation retention priority & 1 & 5 & 9 \\
Scheduling priority & 3 & 4 & 9 \\
Delay budget & $50 \mathrm{~ms}$ & $150 \mathrm{~ms}$ & $300 \mathrm{~ms}$ \\
Packet error loss rate & $10^{-3}$ & $10^{-3}$ & $10^{-6}$ \\
\hline
\end{tabular}

Bearer parameters. LTE defines a class-based QoS provisioning based on the concept of bearers that are used to gather packets that have to receive a common QoS treatment. Two types of bearers are defined: Guaranteed Bit Rate (GBR) bearers and Non-GBR bearers. A bearer is associated to a QoS Class Identifier (QCI) characterized by priority level, packet delay budget and acceptable packet loss rate. In addition, a GBR bearer has a fixed UpLink (UL) and DownLink (DL) data rates. The simulated LTE architecture considers 3 bearers according to the data type [18]. The first one is a GBR bearer 
for TCMS Signalling data with the highest priority and scheduling priority. The second one is also a GBR bearer for TCMS Voice data with a lower priority and scheduling priority. The last one is a non-GBR bearer for TCMS Video and File data and all the Passenger UE data. Table 4 summarizes the bearers' setting.

The train is equipped with the MCG and moves with a high speed $(300 \mathrm{~km} / \mathrm{s})$ back and forth on the defined railway trajectory. During its movement, it exchanges TCMS traffic with the GCG through its connectivity with eNBs. Besides, 8 passengers use the same LTE connectivity to send their application traffic. We use $4 \mathrm{MoU}, 2 \mathrm{MuU}$ and 2 EnU as described in table 3.

\subsection{Performance evaluation}

This part focuses on evaluating whether LTE can provide the required performance in the TCMS traffic exchange between MCG and GCG. For this purpose, we evaluate the data loss and the data transfer delay for TCMS traffics.

Data integrity. This performance allows evaluating the capacity of LTE to transfer TCMS application data between MCG and GCG, when the connection is shared with passengers. Simulation results show that LTE provides good performance. As shown in table 5, all signaling data is successfully transmitted from the MCG to the GCG (Fig.2), thanks to its priority among others and parameters provided from LTE such as the Guaranteed Bearer Rate (GBR) that allows transmission integrity. Besides, this traffic application is transferred through the TCP protocol following the ARQ (Automatic Repeat reQuest) scheme, which allows retransmission of lost and error data. Some fluctuations in the Voice data transmission since it uses UDP protocol without retransmission of lost packets. However, the Video application data is not well transmitted because there is no guarantee parameter used for this traffic kind, as well as the UDP protocol is used for its transferred.

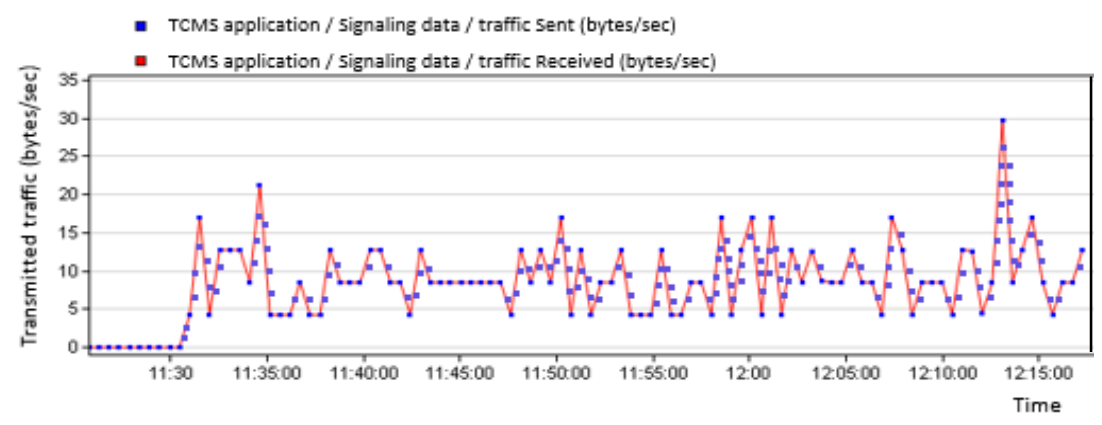

Fig. 2. Sent/received MCG signaling data. 
Table 5. Data delivery ratio for different TCMS applications

\begin{tabular}{llllll}
\hline & $\begin{array}{l}\text { Signaling } \\
\text { data }\end{array}$ & $\begin{array}{l}\text { File update } \\
\text { data }\end{array}$ & $\begin{array}{l}\text { Video } \\
\text { data }\end{array}$ & $\begin{array}{l}\text { Voice data } \\
\text { (live+ Recorded) }\end{array}$ \\
\hline Transfer protocol & TCP & TCP & UDP & UDP \\
\hline Data delivery Ratio & $100 \%$ & $100 \%$ & $62.26 \%$ & $97.4 \%$ \\
\hline
\end{tabular}

Fig. 3 shows dropped packets of the different TCMS traffic detected in the physical LTE layer during the time. At 12:13:00, an important number of data lost is detected due to the scheduling conflicts of the different data at this time that overload links and causes loss of data.

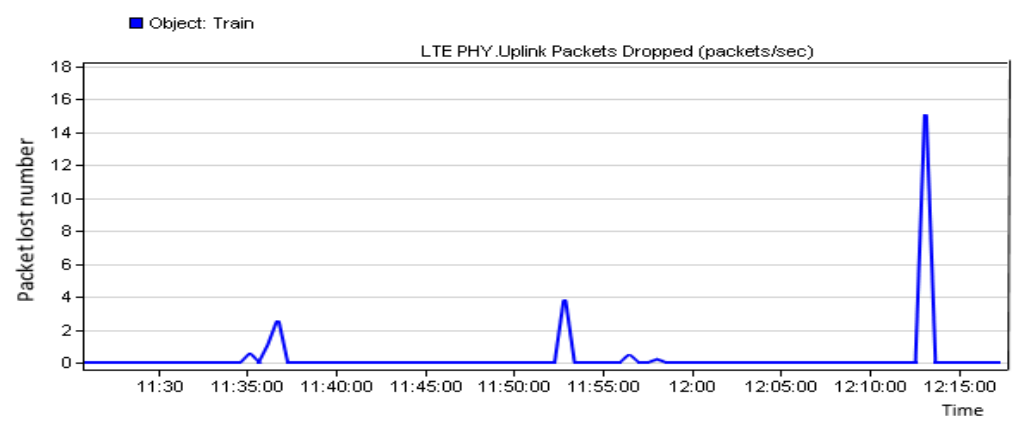

Fig. 3. Packet lost number in the LTE PHY.

Data transfer delay. This performance allows evaluating the required delay to transfer traffic from MCG to GCG. Simulation results show that all application traffic uses a stable delay during the simulation time. Almost traffic delay is around $0.015 \mathrm{sec}$, and only traffic voices take around $0.1 \mathrm{sec}$.

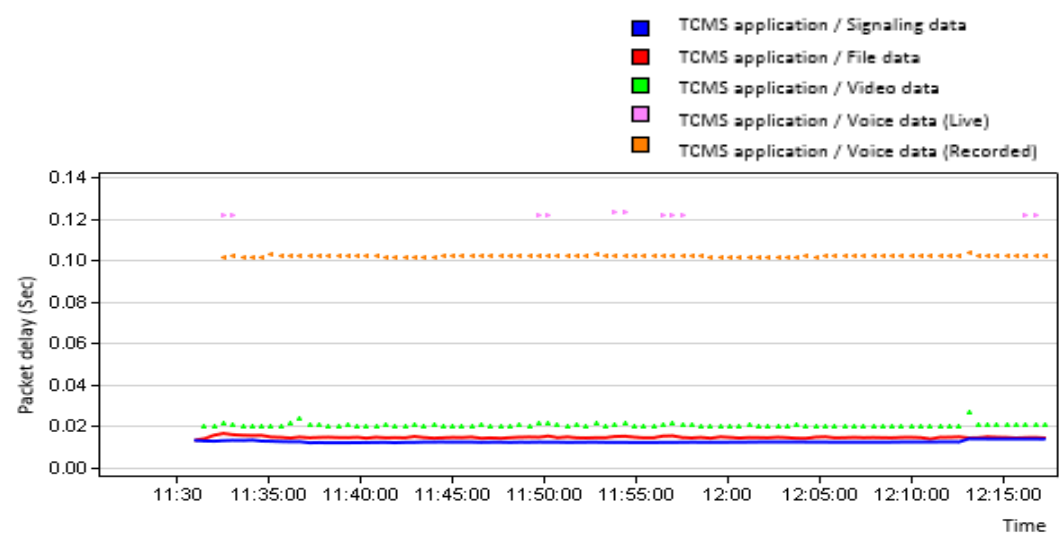

Fig. 4. Packet delay for different TCMS applications. 


\section{Hardware and Software Evaluation Platform for LTE-based Train-to-Ground Communication in the context of TCMS}

As previously mentioned, several authors have consider performance evaluation of LTE network based on pure simulations using Riverbed modeler as in the first part of this paper. However, these simulation tools can only measure network-level metrics for LTE applications and the transmission-level features cannot be examined. Therefore, real-time emulators have been also presented in the literature for the same purposes, e.g. Open Air Interface (OAI) [19], openLTE [20], and srs-LTE [21].

\subsection{HW and SW Co-simulation using System-in-the-loop (SITL)}

Main challenging issues in realizing co-simulation is the need of synchronization of progress or event and conversion of packets between real and simulation environments. The real-time OAI emulator operates at continuous time. On the other hand, the communication network simulator, Riverbed Modeler, is discrete-event driven. In the Safe4Rail project, we solved the problem by using SITL (System In The Loop) module in the network simulator as a gateway to connect and interact between a simulated network running in Riverbed Modeler and a test platform with the external physical hardware in real-time. In the simulation environment, a SITL gateway is used as the interface module to connect the network interface of the host computer.

\subsection{Co-simulation Platform: Architecture, Components and Configurations}

The co-simulation platform has been setup in order to assess the performance and reliability of the LTE network for the TCMS traffic exchange in realistic network architecture and configurations. The developed testbed consists of a real network represented by OAI setup and a simulation model in Riverbed Modeler environment. The architecture of the testbed is shown in Fig. 5.

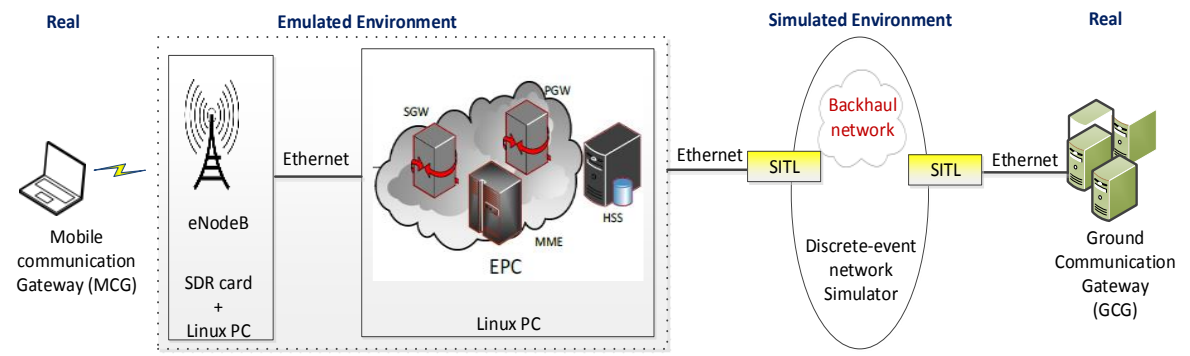

Fig. 5. The proposed co-simulation evaluation testbed.

The OAI setup is dedicated to the emulation of the LTE network behavior. Whereas, the Riverbed Modeler scenario represents communication network that is the backhaul network of railway communication. Then, the OAI emulator is expected to reproduce the Radio Frequency (RF) transmission features, while the communication network 
modelling is a discrete event-based simulation. This solution is more complex than other pure simulated system (as presented in the first part of the paper) or pure real infrastructure due to the combination. Meanwhile, it provides an opportunity to examine the data transmission from an end-to-end point of view.

A brief description of each component and configurations in the proposed framework is as following:

1) OAI LTE emulator: The methodology deployed in the OAI platform is to use the real stack to perform more realistic and reliable simulations. In OAI software implementation for eNB, it provides the entire protocol stack from physical to network layer, e.g. PHY, MAC, RLC, PDCP and RRC. Regarding the core network, the OAI implements the EPC components with the Serving Gateway (SGW), the Packet Data network Gateway (P-GW), the Mobility Management Entity (MME), the Home Subscriber Server (HSS) and the Non-Access Stratum (NAS) protocols [22-23].

2) Riverbed Modeler network simulator: two SITL gateway modules are used at each side connecting to real network hardware. In order to run the simulation correctly and efficiently, the simulator and the SITL gateway node need to be pre-configured. For example, the real-time execution ratio property should be set, so that the simulation time in the simulator is ensured to be consistent with the real time. The SITL gateway node is assigned with the right network card. In addition, the rule for packet filtering is set appropriately in order to reduce the amount of processed data.

3) Application server: the application server is a Linux-based computer in the developed platform. It serves as the server part for TCP/UDP and video traffic.

\section{Test Cases and Results}

\subsection{Testbed Setup}

We illustrate a co-simulation platform for the evaluation of the TCMS traffic exchange between MCG and GCG using the communication technology LTE As shown in Fig. 6, the testbed is placed in our lab environment and there is no other interfering network and disturbing of wireless signals in range during the experiment. The scenario includes an OAI-based LTE emulator connecting to a simple backhaul network model in the Riverbed Modeler via the SITL interface module. The different hardware and software deployed in this project are listed in Table 6. 


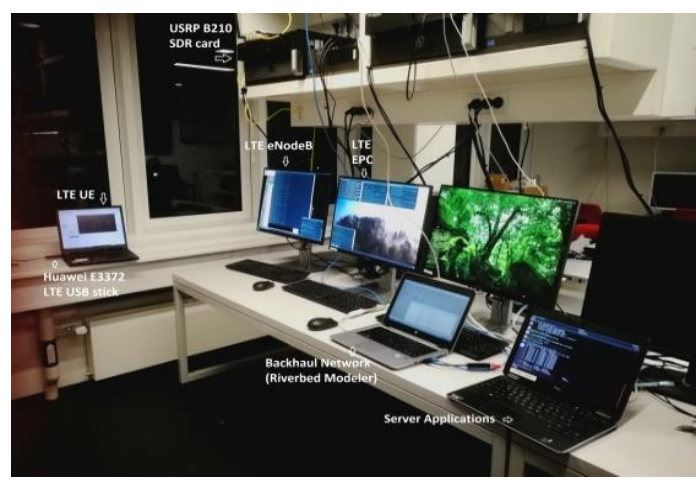

Fig. 6. The proposed co-simulation evaluation testbed.

The LTE emulator consists of a LTE UE, eNB and EPC. LTE eNB is a SDR base station that runs the OAI software and allows the connection of the UE and transforms the LTE frame into an Ethernet frame and vice versa. The SDR solution is based Universal Software Radio Peripheral (USRP) B210 platform, which acts as radio front-end with continuous frequency coverage from $70 \mathrm{MHz}-6 \mathrm{GHz}$. Table 6 lists the RF properties of the LTE eNB in the measurement setup.

Table 6. RF Properties of the test setup

\begin{tabular}{ll}
\hline & LTE eNodeB Properties \\
\hline Frequency band & $2.6 \mathrm{GHz}$, Band 7 \\
Bandwidth & $10 \mathrm{MHz}(50 \mathrm{DL}$ RBs $)$ \\
Duplex mode & FDD (Frequency Division Duplex) \\
\hline
\end{tabular}

\subsection{Measurements for Co-simulation Platform}

The aim of this setup consists in carrying out the performance test. Three different wireless transmissions are configured in the LTE emulator, from good signal strength to bad condition. Using the signal monitoring and reporting from OAI software, the LTE uplink transmission performances are observed and listed in table 7. I/Q constellation maps out the carried uplink data to the UE on Physical Uplink Shared Channel (PUSCH), where the 16-QAM modulation scheme is used. Physical Uplink Control Channel (PUCCH) is used to transmit the uplink control information, such as Scheduling Request (SR) message. UE sends SR message to ask the network to allocate resources so that UE can transmit PUSCH. 
Table 7. LTE uplink transmission performances of the test setup

\begin{tabular}{|c|c|c|c|}
\hline & Signal level 1 & Signal level 2 & Signal level 3 \\
\hline PUSCH I/Q & & & \\
\hline $\begin{array}{l}\text { Power } \\
\text { PUCCH }\end{array}$ & $-77 /-96 \mathrm{dBm}$ & $-78 /-96 \mathrm{dBm}$ & $-94 /-96 \mathrm{dBm}$ \\
\hline $\begin{array}{l}\text { SR received/ } \\
\text { total }\end{array}$ & 987/50434 (diff 49447) & $\begin{array}{l}\text { 1103/56783 (diff } \\
55680)\end{array}$ & $\begin{array}{l}\text { 1200/61621 (diff } \\
60421)\end{array}$ \\
\hline
\end{tabular}

The LTE EPC provides data communication between the LTE network and the GCG (end server). As shown in Fig. 7, the backhaul network in the testbed contains basic network architecture with a single router connecting by two SITL interfaces for packets conversion between the real and simulated environment. The router configurations are chosen such that various traffic load can be inserted in order to study the TCMS traffic shaping and scheduling effect on the received data.

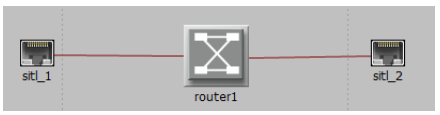

Fig. 7. The proposed co-simulation evaluation testbed.

Table 8. Measurement results of test setup

\begin{tabular}{llll}
\hline & Signal level 1 & Signal level 2 & Signal level 3 \\
\hline TCP data & & & \\
\hline $\begin{array}{l}\text { Transfer data size } \\
\text { Throughput }\end{array}$ & $\begin{array}{l}11.8 \mathrm{MB} \\
9.82 \mathrm{Mbps}\end{array}$ & $\begin{array}{l}2.58 \mathrm{MB} \\
1.98 \mathrm{bps}\end{array}$ & $\begin{array}{l}1.58 \mathrm{MB} \\
1.40 \mathrm{Mbps}\end{array}$ \\
\hline UDP data with length of $\mathbf{1 0 0 0}$ bytes & & \\
\hline Transfer data size & $1.24 \mathrm{MB}$ & $1.24 \mathrm{MB}$ \\
Throughput & $1.04 \mathrm{Mbps}$ & $1.04 \mathrm{Mbps}$ \\
Jitter & $5.234 \mathrm{~ms}$ & & $55.272 \mathrm{~ms}$ \\
Lost/total & $2 \%$ & $69 \%$ \\
\hline Video streaming & & \\
\hline Decoded & 352 blocks & $340 \mathrm{blocks}$ & $341 \mathrm{blocks}$ \\
Played & 808 frames & 767 frames & 703 frames \\
Lost & 0 frames & 5 frames & 18 frames \\
\hline
\end{tabular}


To evaluate the performance of the co-simulation platform, we carried out measurements in a single eNB LTE cellular network and a static UE. The scenario with three levels of signal conditions (from good to worse) is applied in the experiment. Traffic under test is TCP/UDP packets generated by the network testing tool IPERF [24] and video streaming packet produced by VLC Media player [25]. The results of testbed show degraded performances (such as reduced bandwidth, increased jitter, raised loss rate) along with the deterioration in signal quality.

\section{Conclusion}

This paper has presented two complementary strategies to evaluate the TCMS application traffic exchange between MCG and GCG through an LTE network: pure simulation platform and a software and hardware in the loop based platform. Pure simulation platform is based on the use of the discrete-event simulator Riverbed Modeler. Preliminary simulation results for a high-speed scenario showed that LTE can be the alternative technology communication, as it allows the integrity of transferring the interesting application traffic, when passengers may use LTE simultaneously. The software and hardware in the loop based platform consists of a communication system able mutually exchanging data between real network devices and Riverbed Modeler. The interconnection between real equipment and simulation demonstrated a way to examine an endto-end network communication and quality of service assurance. The evaluation offered by both platforms will allow discussing the potential usage of wireless TCMS.

The future work will consist in a deeper study and analyze for other uses cases such as train station and existence of other trains in the parallel lines using the same LTE cell (shunting areas for example or high density lines) and also varying the environmental conditions (hilly terrain, rural, tunnels, etc.). The channel models considered in the LTE configuration will be also changed in order to be closer to railway radio channel conditions.

\section{References}

1. https://www.railengineer.uk/2015/08/11/what-is-tcms/.

2. Roll2Rail Project. Deliverable 2.6: Architecture and Interface Definition for the Train to Ground Communication, 2016.

3. EU. CONNECTA. D2.1: Requirements and Specification for the T2G System. Contributing to Shift2Rail's next generation of high capable and safe TCMS and brakes, CONNECTA, 9.6.2017. http://projects.shift2rail.org/s2r_ip1_n.aspx?p=CONNECTA

4. IEC Standard. IEC 61375-2-6: Electronic Railway equipment train communication network (TCN): Part 2-6: On-board to Ground Communication. IEC, 19.8.2016.

5. [https://shift2rail.org/projects/connecta/]

6. EU. Safe4Rail. D3.2 Report on design of TCMS distributed simulation framework concept (submitted: July 2017, M10) - https://safe4rail.eu/news/deliverables

7. EU. Safe4Rail. D3.3 Report on design of T2G Test Environment (submitted: July 2017, M10) - https://safe4rail.eu/news/deliverables 
8. Bouaziz, M., Yan, Y., Kassab, M., Soler, J., Berbineau, M. Train-to-Ground communications of a Train Control and Monitoring Systems: A simulation platform modelling approach, 7th Transport Research Arena TRA 2018, April 16-19, 2018, Vienna, Austria.

9. Sniady, A., Soler, J., Kassab, M., Berbineau, M., Ensuring Long-Term Data Integrity: ETCS Data Integrity Requirements Can Be Fulfilled Even under Unfavorable Conditions with the Proper LTE Mechanisms, IEEE Vehicular Technology Magazine, 2016, Volume: 11, Issue: 2, Pages: 60 - 70, DOI: 10.1109/MVT.2015.2503882

10. Khayat, A., Kassab, M., Berbineau, M., Abid, M. A., Belghith, A., LTE Based Communication System for Urban Guided-Transport: A QoS Performance Study, Nets4carsNets4trains 2013, 5th workshop on Communication Technologies for Vehicles, Nets4cars, 14/05/2013, Villeneuve d'Ascq, FRANCE, 2013

11. Aguado, M., Jacob, E., Berbineau, M., Astorga, J., Toledo, N., The cross layer RMPA handover: a reliable mobility pattern aware handover strategy for broadband wireless communication in a high-speed railway domain, EURASIP Journal on Wireless Communications and Networking 2012, 2012:298 doi:10.1186/1687-1499-2012-298

12. Sniady A., Soler J. Performance of LTE in High Speed Railway Scenarios. Communication Technologies for Vehicles. Nets4Cars/Nets4Trains 2013. Lecture Notes in Computer Science, vol 7865. Springer, Berlin, Heidelberg.

13. Riverbed modeler, 2017. www.riverbed.com.

14. Modeler Documentation Set, version 18.6, revised 9/30/16, ( 2016 Riverbed Technology.

15. Sniady, A., Soler, J., and Dittmann, L. 2015. Communication Technologies Support to Railway Infrastructure and Operations. Ph.D. thesis, DTU Fotonik, 2015, DOI: 10.11581/DTU:00000010.

16. K. Guan, Z. Zhong, and B. Ai, "Assessment of LTE-R using High Speed Railway Channel Model," in Proceedings of the 3rd International Conference on Communications and Mobile Computing (CMC), pp. 461-464, IEEE, 2011. ISBN 978-1-61284-312-4.

17. http://www.roll2rail.eu/

18. 3rd Generation Partnership Project, Technical Specification Group Services and System Aspects, Policy and charging control architecture (Release 10), march 2012.

19. N. Nikaein, R. Knopp, F. Kaltenberger, L. Gauthier, C. Bonnet, D. Nussbaum,and R. Ghaddab, "Demo: OpenAirInterface: An open LTE network in a PC," in Proceedings of the 20th Annual International Conference on Mobile Computing and Networking, ser. MobiCom '14. New York, NY, USA: ACM, 2014, pp. 305-308.

20. B. Wojtowicz, “OpenLTE,” jan. 2017. [Online]. Available: http: //openlte.sourceforge.net/

21. I. Gomez-Miguelez, A. Garcia-Saavedra, P. D. Sutton, P. Serrano, C. Cano, and D. J. Leith, "srsLTE: An open-source platform for LTE evolution and experimentation," in Proceedings of the Tenth ACM International Workshop on Wireless Network Testbeds, Experimental Evaluation, and Characterization, ser. WiNTECH '16. New York, NY, USA: ACM, 2016, pp. 25-32.

22. Open air interface Homepage, http://www.openairinterface.org, last accessed 2017/12/12

23. Hicham Anouar, Christian Bonnet, Daniel Câmara, Fethi Filali, Raymond Knopp, An overview of OpenAirInterface wireless network emulation methodology, ACM SIGMETRICS Performance Evaluation Review, v.36 n.2, September 2008

24. iperf homepage, https://iperf.fr/

25. VLC media player, homepage, https://www.videolan.org/vlc/index.html 